\title{
SOME INEQUALITIES FOR POLYNOMIALS
}

\author{
Q. I. RAHMAN
}

\begin{abstract}
Let $p_{n}(z)$ be a polynomial of degree $n$. Given that $p_{n}(z)$ has a zero on the circle $|z|=\rho(0<\rho<\infty)$ we estimate $\max _{|z|=R>1}\left|p_{n}(z)\right|$ in terms of $\max _{|z|=1}\left|p_{n}(z)\right|$. We also consider some other related problems.
\end{abstract}

It is well known (see [8, p. 346], or [6, vol. 1, p. 137, Problem III 269]) that if $p_{n}(z)=\sum_{k=0}^{n} a_{k} z^{k}$ is a polynomial of degree $n$ such that $\left|p_{n}(z)\right|$ $\leqq M$ for $|z| \leqq 1$, then at a point $z$ outside the unit disk

$$
\left|p_{n}(z)\right| \leqq M|z|^{n}
$$

where equality holds at some point $z_{0}$ with $\left|z_{0}\right|>1$ only if it holds at all such points and that is possible only when $p_{n}(z)=a_{n} z^{n}=M e^{i \gamma} z^{n}$, i.e. when all the zeros of $p_{n}(z)$ lie at the origin. It is therefore natural to ask what improvement results from supposing that $p_{n}(z)$ has a zero of modulus $\rho$. We have recently proved that in case $\rho=1$, we may replace (1) by ([4], see $\left.\left(1.7^{\prime \prime}\right)\right)$

$$
\max _{|z|=R>1}\left|p_{n}(z)\right| \leqq M R^{n}\left\{1-\frac{2-\sqrt{ } 2}{2 n}\left(1-R^{-1}\right)^{2}\right\}
$$

The proof of (2) depended very much on the fact that the prescribed zero was located on $|z|=1$, and could not be modified in any obvious way to deal with the problem in its full generality. Here we prove:

THEOREM 1. Let $p_{n}(z)$ be a polynomial of degree $n$ having a zero of modulus $\rho$ $(0<\rho<\infty)$, and satisfying $\left|p_{n}(z)\right| \leqq M$ for $|z| \leqq 1$. Denote by $\sigma_{n}$ and $\tau_{n}$ the smallest positive roots of the equations

$$
x^{n+1}-2 x+1=0
$$

and

$$
(n+1) x^{n+2}-(n+3) x^{n+1}+(n+1) x-(n-1)=0,
$$

respectively. Then

$$
\begin{aligned}
\max _{|z|=R>1}\left|p_{n}(z)\right| & \leqq \frac{d(\rho) R+M}{M R+d(\rho)} M R^{n} \\
& \leqq d(\rho) R^{n}+\frac{M^{2}-(d(\rho))^{2}}{M} R^{n-1}
\end{aligned}
$$

Received by the editors October 4, 1974.

AMS (MOS) subject classifications (1970). Primary 30A06, 30A64; Secondary 26A82.

Key words and phrases. Polynomials with a prescribed zero, Schwarz's lemma. 
where

$$
d(\rho)= \begin{cases}1-\frac{1-\rho}{1+\rho} \frac{\rho^{n}}{1-\rho^{n}} & \text { if } 0 \leqq \rho \leqq \sigma_{n}, \\ 1-\frac{1-\rho}{1+\rho} \frac{2 \rho^{n+1}}{1-\rho^{n+1}} & \text { if } \sigma_{n} \leqq \rho \leqq \tau_{n}, \\ \frac{n}{n+1} \frac{2}{1+\rho} & \text { if } \tau_{n} \leqq \rho \leqq 1, \\ \frac{1}{\rho} d\left(\frac{1}{\rho}\right) & \text { if } 1 \leqq \rho .\end{cases}
$$

It may be noted that (5) not only extends but also refines (2).

Theorem 1 is an immediate consequence of Lemmas 1 and 2 below.

LEMMA 1. If $p_{n}(z)=\sum_{k=0}^{n} a_{k} z^{k}$ is a polynomial of degree $n$ having a zero of modulus $\rho$ then

$$
\left|a_{n}\right| \leqq d(\rho) \max _{|z|=1}\left|p_{n}(z)\right|
$$

where $d(\rho)$ is given by (6). For small as well as large values of $\rho$ the inequality is essentially best possible.

Lemma 1 is readily obtained on applying the following result $[7$, Theorem 3] to the polynomial $z^{n} p(1 / z)$.

THEOREM A. Let $p_{n}(z)=\sum_{k=0}^{n} a_{k} z^{k}$ be a polynomial of degree $n$ having a zero of modulus $\rho$. If $\sigma_{n}$ and $\tau_{n}$ denote the smallest positive roots of (3) and (4) respectively, then

$$
\left|a_{0}\right| \leqq c(\rho) \max _{|z|=1}\left|p_{n}(z)\right|
$$

where

$$
c(\rho)= \begin{cases}\rho-\frac{1-\rho}{1+\rho} \frac{\rho^{n+1}}{1-\rho^{n}} & \text { if } 0 \leqq \rho \leqq \sigma_{n}, \\ \rho-\frac{1-\rho}{1+\rho} \frac{2 \rho^{n+2}}{1-\rho^{n+1}} & \text { if } \sigma_{n} \leqq \rho \leqq \tau_{n}, \\ \frac{n}{n+1} \frac{2 \rho}{1+\rho} & \text { if } \tau_{n} \leqq \rho \leqq 1, \\ \rho c(1 / \rho) & \text { if } 1 \leqq \rho .\end{cases}
$$

The estimate is essentially best possible for small as well as for large values of $\rho$.

LeMmA 2. If $p_{n}(z)=\sum_{k=0}^{n} a_{k} z^{k}$ is a polynomial of degree $n$ such that $\max _{|z|=1}\left|p_{n}(z)\right| \leqq M$, then for $|z|=R>1$,

$$
\left|p_{n}(z)\right| \leqq \frac{\left|a_{n}\right| R+M}{M R+\left|a_{n}\right|} M R^{n} ;
$$

a fortiori, we obtain 


$$
\left|p_{n}(z)\right| \leqq\left|a_{n}\right| R^{n}+\frac{M^{2}-\left|a_{n}\right|^{2}}{M} R^{n-1} \text {. }
$$

Proof of Lemma 2. It is clear that $q(z) \equiv z^{n} p_{n}(1 / z)$ is also a polynomial. Besides,

$$
\left|q\left(e^{i \theta}\right)\right| \equiv\left|p_{n}\left(e^{-i \theta}\right)\right| \quad \text { for real } \boldsymbol{\theta} .
$$

Hence $\max _{|z|=1}|q(z)|=\max _{|z|=1}\left|p_{n}(z)\right| \leqq M$ and by a well-known generalization of Schwarz's lemma (see for example [5, p. 167])

$$
|q(z)| \leqq M \frac{M|z|+|q(0)|}{|q(0)||z|+M}=M \frac{M|z|+\left|a_{n}\right|}{\left|a_{n}\right||z|+M} \text { for }|z|<1 .
$$

Replacing $z$ by $1 / z$ we obtain the desired result.

ReMARK. We observe that $\left|a_{n}\right|,\left(M^{2}-\left|a_{n}\right|^{2}\right) / M$ appearing on the righthand side of $\left(8^{\prime}\right)$ cannot in general be replaced by smaller numbers. Given $\varepsilon>0$ we construct polynomials $p_{n}(z)=\sum_{k=0}^{n} a_{k} z^{k}$ of degree $n>(2 / \varepsilon)-1$ with

$$
\begin{gathered}
\max _{|z|=1}\left|p_{n}(z)\right| \leqq M \text { and } \\
\max _{|z|=R}\left|p_{n}(z)\right|>\left|a_{n}\right| R^{n}+\left(\frac{M^{2}-\left|a_{n}\right|^{2}}{M}-\varepsilon\right) R^{n-1} \text { for } R>\frac{M \sqrt{ } n}{\varepsilon} .
\end{gathered}
$$

Let $0<|\alpha|<M$ and consider the function

$$
w=f(z)=M \frac{M z+\alpha}{\bar{\alpha} z+M}=\alpha+\frac{M^{2}-|\alpha|^{2}}{M} z+\sum_{k=2}^{\infty} c_{k} z^{k},
$$

which is analytic in $|z|<M /|\alpha|$ and maps the closed unit disk onto the disk $|w| \leqq M$. If

$$
\sigma_{n}(z)=\frac{s_{0}(z)+s_{1}(z)+\cdots+s_{n}(z)}{n+1}
$$

where $s_{0}(z), s_{1}(z), \ldots, s_{n}(z), \ldots$ are the partial sums of the Taylor series of $f(z)$ then $[9$, p. 236]

$$
\max _{|z|=1}\left|\sigma_{n}(z)\right| \leqq M \quad \text { for } n=0,1,2, \ldots
$$

Hence

$$
\begin{aligned}
p_{n}(z) & =z^{n} \sigma_{n}(1 / z) \\
& =\alpha z^{n}+\frac{M^{2}-|\alpha|^{2}}{M} \frac{n}{n+1} z^{n-1}+\sum_{k=2}^{n} \frac{n-k+1}{n+1} c_{k} z^{n-k}
\end{aligned}
$$

is a polynomial of degree $n$ with

$$
\left|p_{n}(z)\right| \leqq M \text { for }|z|=1
$$

Since 


$$
|\alpha|^{2}+\left(\frac{M^{2}-|\alpha|^{2}}{M}\right)^{2}+\sum_{k=2}^{\infty}\left|c_{k}\right|^{2}=\frac{1}{2 \pi} \int_{0}^{2 \pi}\left|f\left(e^{i \theta}\right)\right|^{2} d \theta \leqq M^{2}
$$

we have

$$
\sum_{k=2}^{n}\left|c_{k}\right|^{2} \leqq \frac{|\alpha|}{M}\left(M^{2}-|\alpha|^{2}\right)^{1 / 2} \leqq \frac{M}{2}
$$

and therefore

$$
\begin{aligned}
\max _{|z|=R}\left|p_{n}(z)\right| \geqq & \max _{|z|=R}\left|\alpha z^{n}+\frac{M^{2}-|\alpha|^{2}}{M} \frac{n}{n+1} z^{n-1}\right| \\
& -\sum_{k=2}^{n} \frac{n-k+1}{n+1}\left|c_{k}\right| R^{n-k} \\
\geqq & |\alpha| R^{n}+\frac{M^{2}-|\alpha|^{2}}{M} \frac{n}{n+1} R^{n-1} \\
& -\left(\sum_{k=2}^{n}\left(\frac{n-k+1}{n+1}\right)^{2} R^{2 n-2 k}\right)^{1 / 2}\left(\sum_{k=2}^{n}\left|c_{k}\right|^{2}\right)^{1 / 2} \\
> & |\alpha| R^{n}+\left\{\frac{M^{2}-|\alpha|^{2}}{M}-\left(\frac{1}{n+1} \frac{M^{2}-|\alpha|^{2}}{M}+\frac{M}{2} \sqrt{ } n \frac{1}{R}\right)\right\} R^{n-1} \\
> & |\alpha| R^{n}+\left(\frac{M^{2}-|\alpha|^{2}}{M}-\varepsilon\right) R^{n-1}
\end{aligned}
$$

if $n>(2 / \varepsilon)-1$ and $R>(M \bigvee n) / \varepsilon$.

As mentioned earlier, equality holds in (1) only when the coefficients $a_{0}, a_{1}, \ldots, a_{n-1}$ are all zero. From Lemma 2 we deduce

THeOREM 2. If $p_{n}(z)=\sum_{k=0}^{n} a_{k} z^{k}$ is a polynomial of degree $n$ such that $\max _{|z|=1}\left|p_{n}(z)\right| \leqq M$ and $\max _{0 \leqq k \leqq n-1}\left|a_{k}\right|=a(0 \leqq a \leqq M)$, then for $|z|$ $=R>1$,

$$
\begin{aligned}
\left|p_{n}(z)\right| & \leqq \frac{\left(M^{2}-M a\right)^{1 / 2} R+M}{M R+\left(M^{2}-M a\right)^{1 / 2}} M R^{n} \\
& \leqq\left(M^{2}-M a\right)^{1 / 2} R^{n}+a R^{n-1} .
\end{aligned}
$$

Proof. If $f(z)=\sum_{k=0}^{\infty} c_{k} z^{k}$ is analytic in $|z|<1$ where $|f(z)| \leqq M$ then ([5, p. 172], see Exercise 9)

$$
\left|c_{0}\right|^{2}+\left|c_{k}\right| M \leqq M^{2}, \quad k=1,2, \ldots
$$

Applying this result to the function

$$
z^{n} p_{n}(1 / z)=a_{n}+a_{n-1} z+\cdots+a_{n-k} z^{k}+\cdots+a_{0} z^{n}
$$

we obtain $\left|a_{n}\right| \leqq\left(M^{2}-M a\right)^{1 / 2}$ and then (9) follows from Lemma 2 .

A theorem of van der Corput and Visser [3] says that if $p_{n}(z)=\sum_{k=0}^{n} a_{k} z^{k}$ is a polynomial of degree $n$ such that $\max _{|z|=1}\left|p_{n}(z)\right| \leqq M$ and $a_{u}, a_{v}(u<v)$ 
are two coefficients such that for no other coefficient $a_{w} \neq 0$ we have $w \equiv u \bmod (v-u)$, then

$$
\left|a_{u}\right|+\left|a_{v}\right| \leqq M
$$

Hence, in particular

$$
\left|a_{n}\right| \leqq M-\max _{0 \leqq k \leqq(n+1) / 2}\left|a_{k}\right|
$$

and as another application of Lemma 2 we obtain

THeOREM 3. If $p_{n}(z)=\sum_{k=0}^{n} a_{k} z^{k}$ is a polynomial of degree $n$ such that $\max _{|z|=1}\left|p_{n}(z)\right| \leqq M$ and $\max _{0 \leqq k \leqq(n+1) / 2}\left|a_{k}\right|=b(0 \leqq b \leqq M)$, then for $|z|$ $=R>1$

$$
\left|p_{n}(z)\right| \leqq \frac{(M-b) R+M}{M R+(M-b)} M R^{n} .
$$

REMARK. It follows from (11) that if $p_{n}(z)=\sum_{k=0}^{n} a_{k} z^{k}$ is a polynomial of degree $n$ such that $\left|a_{0}\right| \geqq\left|a_{n}\right|$ then $\left|a_{n}\right| \leqq \frac{1}{2} \max _{|z|=1}\left|p_{n}(z)\right|$, and by Lemma 2

$$
\begin{aligned}
\max _{|z|=R>1}\left|p_{n}(z)\right| & \leqq \frac{(R / 2)+1}{R+(1 / 2)} R^{n} \max _{|z|=1}\left|p_{n}(z)\right| \\
& \leqq\left(\frac{1}{2} R^{n}+\frac{3}{4} R^{n-1}\right) \max _{|z|=1}\left|p_{n}(z)\right| .
\end{aligned}
$$

The condition $\left|a_{0}\right| \geqq\left|a_{n}\right|$ is satisfied if for example $p_{n}(z)$ has all its zeros in $|z| \geqq 1$ or $p_{n}(z)$ is self reciprocal, i.e. $z^{n} \overline{p_{n}(1 / \bar{z})} \equiv p_{n}(z)$. But in these two cases (13) can be replaced by the much stronger (and sharp) inequality ([1], [2])

$$
\max _{|z|=R>1}\left|p_{n}(z)\right| \leqq \frac{R^{n}+1}{2} \max _{|z|=1}\left|p_{n}(z)\right| .
$$

Inequality (13) holds also for polynomials $p_{n}(z)$ for which $z^{n} p_{n}(1 / z) \equiv p_{n}(z)$. However, we do not know the precise estimate in this case. It is readily seen that (14) holds if $n=1$. We can show that it also holds if $n=2$. In fact, if $p_{2}(z)=a_{2} z^{2}+a_{1} z+a_{0}$ is such that $z^{2} p_{2}(1 / z) \equiv p_{2}(z)$ then $a_{2}=a_{0}$ and

$$
\begin{aligned}
\frac{\max _{|z|=R>1}\left|p_{2}(z)\right|}{\max _{|z|=1}\left|p_{2}(z)\right|} & =R \frac{\max _{|z|=R>1}\left|a_{2}(z+1 / z)+a_{1}\right|}{\max _{|z|=1}\left|a_{2}(z+1 / z)+a_{1}\right|} \\
& =R \max _{w \in \mathcal{E}}\left|2 a_{2} w+a_{1}\right| / \max _{w \in[-1,1]}\left|2 a_{2} w+a_{1}\right|
\end{aligned}
$$

where $\varepsilon$ is the ellipse with foci at $1,-1$ and semiaxes $\frac{1}{2}(R+1 / R), \frac{1}{2}(R-1 / R)$. Hence it is enough to show that for an arbitrary complex number $\zeta$

$$
\max _{w \in \mathcal{E}}|w-\zeta| / \max _{w \in[-1,1]}|w-\zeta| \leqq \frac{1}{2}\left(R+\frac{1}{R}\right) .
$$

Clearly, there is no loss of generality in assuming that $\zeta$ lies in the right halfplane $H_{1}$, i.e. $\operatorname{Re} \zeta \geqq 0$. Thus we will like to show that for all $\zeta$ lying in $H_{1}$,

$$
\max _{w \in \mathscr{E}}\left|\frac{w-\zeta}{1+\zeta}\right| \leqq \frac{1}{2}\left(R+\frac{1}{R}\right) \text {. }
$$


Now, let $w=u+i v$ be an arbitrary given point on $\mathcal{E}$. As a function of $\zeta$, $(w-\zeta) /(1+\zeta)$ is analytic except at the point -1 . Hence

$$
\max _{\zeta \in H_{1}}|(w-\zeta) /(1+\zeta)|
$$

cannot be attained at an interior point of $H_{1}$. Therefore, all we need to show is that

$$
\frac{|u+i v-i \eta|}{\sqrt{ }\left(1+\eta^{2}\right)} \leqq \frac{1}{2}\left(R+\frac{1}{R}\right) \text { for } u+i v \in \mathcal{E},-\infty<\eta<\infty .
$$

But this is a matter of simple verification, and hence

$$
\max _{|z|=R>1}\left|p_{2}(z)\right| \leqq \frac{R^{2}+1}{2} \max _{|z|=1}\left|p_{2}(z)\right|
$$

if $z^{2} p_{2}(1 / z) \equiv p_{2}(z)$

\section{REFERENCES}

1. N. C. Ankeny and T. J. Rivlin, On a theorem of S. Bernstein, Pacific J. Math. 5 (1955), 849-852. MR 17, 833.

2. R. P. Boas, Jr. and Q. I. Rahman, $L^{p}$ inequalities for polynomials and entire functions, Arch. Rational Mech. Anal. 11 (1962), 34-39. MR 28 \#2214.

3. J. G. van der Corput and C. Visser, Inequalities concerning polynomials and trigonometric polynomials, Nederl. Akad. Wetensch. Proc. 49, 383-392 = Indag. Math. 8 (1946), 238-247. MR 8, 148.

4. A. Giroux and Q. I. Rahman, Inequalities for polynomials with a prescribed zero, Trans. Amer. Math. Soc. 193 (1974), 67-98.

5. Z. Nehari, Conformal mapping, McGraw-Hill, New York, 1952. MR 13, 640.

6. G. Pólya and G. Szegö, Aufgaben und Lehrsätze aus der Analysis, Springer, Berlin, 1925.

7. Q. I. Rahman and G. Schmeisser, Some inequalities for polynomials with a prescribed zero, Trans. Amer. Math. Soc. 216 (1976), 91-103.

8. M. Riesz, Uber einen Satz des Herrn Serge Bernstein, Acta Math. 40 (1916), 337-347.

9. E. C. Titchmarsh, The theory of functions, 2nd ed., Oxford Univ. Press, Oxford, 1939.

Department of Mathematics, University of Montreal, Montreal, Quebec, Canada 